ISSN: 2572-2964

\title{
TREATMENT OF GENU VALGUM IN CHILDREN METHOD OF TEMPORARY HEMIEPIPHYSIODESIS USING EIGHT- PLATES: SHORT-TERM OUTCOME
}

\author{
Emad Abd-Elhady MD; and Mohamed El-Sadek MD² \\ ${ }^{1}$ Assistant Professor Orthopedic Surgery Department, Zagazig University, Egypt. \\ ${ }^{2}$ Assistant Professor Orthopedic Surgery Department, Zagazig University, Egypt.
}

Abstract:

Background: Angular deformities of the lower extremities cause activity-related pain, altered gait mechanics, and joint instability, and these are exacerbated by periods of growth. Conservative usually fails to correct deformity; correction of deformity by hemiepiphysiodesis has become the standard surgical technique of treatment.

Objective: The aim of this study to evaluate temporary hemiepiphysiodesis around the knee to modulate growth with eight-Plate technique in patient with genu valgum.

Material and method: A prospective study between January 2015 and December 2019, we evaluated the patients as a single group to assess the effectiveness of temporary hemiepiphysiodesis as a method of correction of genu valgum. The study included 29 patients with one patient was male and had unilateral valgus left knee and was lost in the follow up after removal of the plate and the remaining 28 patients were 22 bilateral $78.6 \%$ and 6 unilateral $22.4 \%$ (50 knees) four patients with right side14.3\% and two patient left side $7.1 \%$ with coronal genu valgum deformity indicated for surgical treatment. There were 19 boys (67.8\%) and 9 girls (32.2\%) and all of them were treated by temporary hemiepiphysiodesis using eight-plate technique. The mean average age of the children at a time of the operation was 7.8 2.3 years (range, 4-12.5 years).

Result: The entire patient had complete correction of the deformity. The mean follow-up was $31 \pm 7.8$ months (range 20-48 months) after plate removal. Two patients reached skeletal maturity at the final follow up. The eightplates were inserted on the medial distal femur (MDF) in 6 patients (21.4\%), in proximal tibia (MPT) in 6 (21.4\%) patients and on both femur and tibia (MPT+MDF) in 16 patients (57.2\%). All patients achieved complete deformity correction after plate removal mean duration 19 \pm 4.6 months (14-29 months) and the mean Intermalleolar distances before the procedure was $19.2 \pm 4.3$ changed postoperative to $1.2 \pm 1$ with P. value $>0.001$ and the mean mLDFA changed from $79.2 \pm 5$ to $87.2 \pm 1.5$ and the P. value $>0.001$ after the procedure.

Conclusion: Temporary hemiepiphysiodesis using eight-Plates is a delicate, straight forward and successful strategy used to treat genu valgum by modulating growth. Slight overcorrection is alluring because of the rebound phenomenon, particularly in young patients with high development potential and risk groups, for example, obese children.

Key words: Hemiepiphysiodesis, Eight-plate, Genu valgum, Growth modulation

\section{INTRODUCTION}

Pathologic genu valgum can be either an idiopathic, present terrible due on physeal harm and development capture, metabolic, neuromuscular disarranges, after diseases acquired scatters. One-sided valgus deformation as a rule had a basic reason as, tumor, disease, old malunited crack at the distal femur or proximal tibia, old physeal injury, old 
orthopedic medical procedure, metaphyseal dysplasia, fibular hemimelia, or numerous epiphyseal dysplasia.(1) The closure of distal end of Femur in male usually at 18-19 years of age, and in females had epiphyseal closure at 16-17 years age. In proximal end of Tibia male had epiphyseal closure at 17-18 years age however in females had epiphyseal closure at 16-17 years age.(2) In children the knee changed from varus knee and decrease till it is reversed to valgus knee and increase to $10-15^{\circ}$ valgus degrees at the age of four years then it starts to decrease over next two years reaching adult level ( 7 degrees valgus). $(3,4)$

In 2004, Stevens suggested the use of two-screw plates to temporarily block the bone growth plate. This method involves the placement of an extraperiosteal plate at the level of the particular growth plate segment, either at the summit or in the plane of the deformity.(5) This method was prospectively described as the "eight-plate guided growth method" According to most authors who have applied this method, its advantages relative to staples and transphyseal screws include minimal invasiveness, higher accuracy, greater comfort, better reliability, and improved efficiency as well as an insignificant risk of complications.(6)

Radiographic evaluation was including measurement of the lateral distal femoral angle (LDFA), anatomic femoral tibial angle (FTA), mechanical axis (MA) passing through center of the femoral head to center of the ankle and its deviation in relation to knee center. Clinical examination of patients including type of gait, discrepancy in limb length of lower limbs, patellofemoral joint for alignment and stability, presence of ligament laxity ,presence of chondromalacia patellae, increase Q-angle ,and contracture of iliotibial band in paralytic disorders (7).

Non-surgical treatment of genu valgum indicated in cases with femurotibial angle less than 15 degrees valgus by bracing and follow up which usually non satisfactory in pathological cases of genu valgum. The surgical treatment include two surgical methods either hemiepiphysiodesis using staples or plate and screws, and transphyseal screws percutaneous which indicated in patients more than 15-20 degree of valgus in children less than 10 years of age (8). The other surgical technique is distal femoral varus osteotomy either medial closing or lateral opening wedge osteotomy, medial transverse osteotomy with hazards of complications nonunion and Peroneal nerve injury (9).

Different methods of around knee osteotomy and may be revised for correction of genu valgum deformities in growing children as deformity may recur. Complications after knee osteotomies presented as knee pain, stiffness, non-union, malunion and delayed mobilization. Disadvantages and complications of osteotomies can be decreased or prevented by using the Guided growth plate method for correction of deformities which is a flexible construct of 8 plate and two screws aiming for hemiepiphysiodesis by using safe and effective surgical technique for correction such angular deformity.(10)

Inclusion criteria in this study was skeletally immature patients with coronal plane deformity around the knee with genu valgum either idiopathic or pathological causes. Exclusion criteria were skeletally mature children, children's age less than two years before physeal closure and skeletal maturity, physiologic genu valgum deformity and valgum deformity due to ligamentous laxity and soft tissue imbalance presented in neuromuscular diseases.

\section{Patients And Methods}

From January 2015 and December 2019, we evaluated the patients as a single group to assess the effectiveness of temporary hemiepiphysiodesis as a method of correction of genu valgum. The included 28 patients were 22 bilateral $78.6 \%$ and 6 unilateral $22.4 \%$ (50 knees) four patients with right side $14.3 \%$ and two patient left side $7.1 \%$ with coronal genu valgum deformity indicated for surgical treatment. There were 19 boys $(67.8 \%)$ and 9 girls $(32.2 \%)$ and all of

American Research Journal of Orthopedics and Traumatology 
them were treated by temporary hemiepiphysiodesis using eight-plate technique. The mean average age of the children at a time of the operation was $7.8 \pm 2.3$ years (range, $4-12.5$ years) (table 1 ).

They were treated by temporary hemiepiphysiodesis method using the eight-plate (8-plate) technique using two holes one third tubular plate with $3.5-\mathrm{mm}$ cannulated screws.

The inclusion criteria of this study was one or more of the following criteria: first, an overall coronal plane deformity valgus $\geq 20$ as per Tibiofemoral angle (TFA) that is persistent or worsening over the past 6 months, second, a mechanical axis deviation (MAD) bisecting the knee outside the central one-third of the transverse diameter of the proximal tibial epiphysis, and third, a clinically symptomatic gait impairment in the form of troublesome circumduction gait in association with genu valgum or frequent falls. Exclusions criteria were previous guided growth surgery or osteotomy on the affected limb, osteochondrodysplasias, endocrinopathies, and infectious deformities, and all forms of resistant rickets. The study was approved by our institution's Ethical Committee of Scientific Research.

\section{Preoperative Assessment}

The patient's clinical evaluation included limb lengths measurement, measuring of rotational and angular deformities with evaluation of the gait. The patellar tracking and any ligamentous laxity were recorded. Children with clinically obvious knock knees and an enlarged Intermalleolar distance $(>10 \mathrm{~cm})$ and with increasing valgus despite conservative therapy had their legs X-rayed anterior-posterior standing up. The determination of the Intermalleolar distance to $>10$ $\mathrm{cm}$ is an internal clinical standard which has proved itself for years. It serves as an orientation and is shared with the increasing valgus deformity in conservative therapy.

The mechanical axis deviation (MAD) from the knee joint was recorded for all cases. It was drawn from the center of the hip to the center of the ankle passing by the knee joint as shown in (Fig 1).

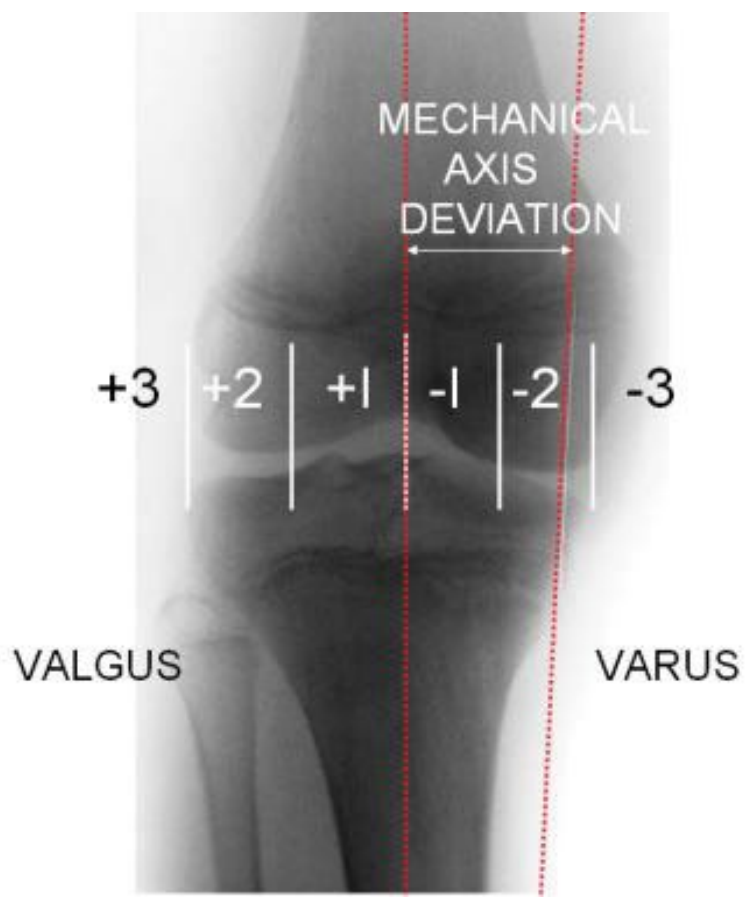

Figure1. Zones of mechanical axisdeviation of knee in angular deformity 
As the mechanical axis normally bisecting the knee, the knee is divided into 4 zones when zone 1 is normal, if the mechanical axis displaced into zone 2 or 3 it is an indication for intervention, especially when the deformity is progressive and symptomatic. To determine the origin of the deformity (femoral, tibial or combined), the mechanical lateral distal femoral angle (mLDFA) and the medial proximal tibial angle (MPTA) were measured for all patients as shown in (Fig 2).

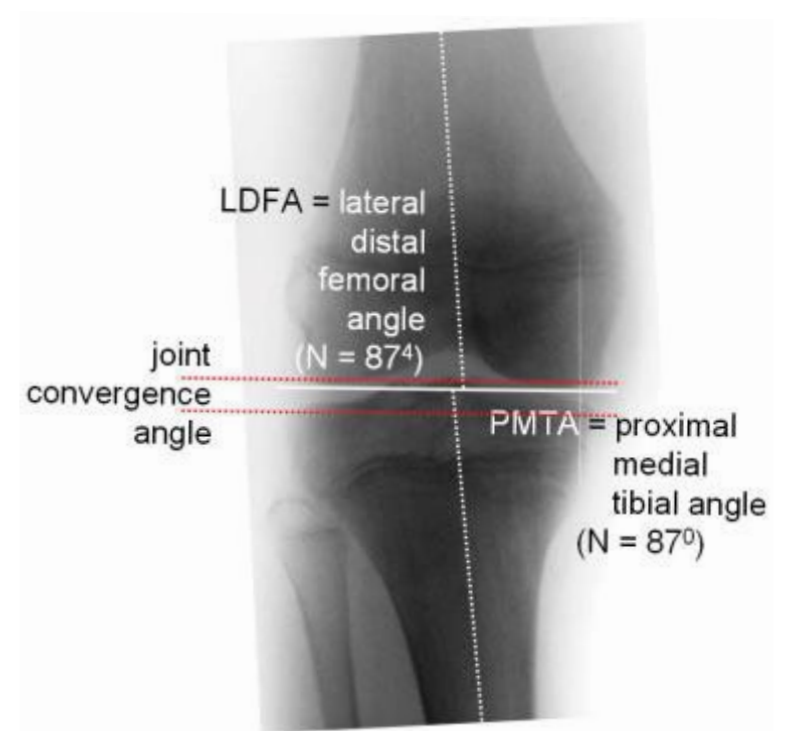

Figure 2. Lateral distal femoral angle(LDFA),proximal medial tibial angle(PMTA)

When indicated, the multiplier method was used to determine if there is sufficient predicted growth remaining to achieve the desired correction through guided growth.

In the event of pathological angles, an assessment was carried out as to whether medial distal femoral and/or medial proximal tibial hemiepiphysiodesis or both should be carried out.(11)

\section{Operative Technique}

As a day case surgery, all cases were operated on under general anesthesia, in the supine position with the application of a tourniquet of the affected side. Under C-Arm fluoroscopic radiation, $3 \mathrm{~cm}$ long skin incision opposite the physis was done medially then the dissection was done through the fascia, between muscles, without elevating the periosteum. To localize the physis, a needle was inserted through the perichondrial ring under fluoroscopy. The plate of 12 or $16 \mathrm{~mm}$ was chosen, then threaded guide pins were inserted through the centers of holes of the plate. A cannulated drill bit of $3.2 \mathrm{~mm}$ caliber was used over the guide wire, and then two $4.5-\mathrm{mm}$ self-tapping cannulated screws were applied with lengths of the screw does not pass the midline. After securing the screws, the wound was closed and the compression bandage was applied.

Starting rehabilitation on the second postoperative day including quadriceps strengthening exercise, increasing range of motion of the knee and starting walking as tolerated by the children. After removal of stitches, full weight bearing was started. After 2 weeks of operation full activity was allowed. Follow up every 2 month till reaching the final correction of the deformity and restoration of normal mechanical axis of the affected limbs. At this time the plate and screws was removed.(fig. 3) 

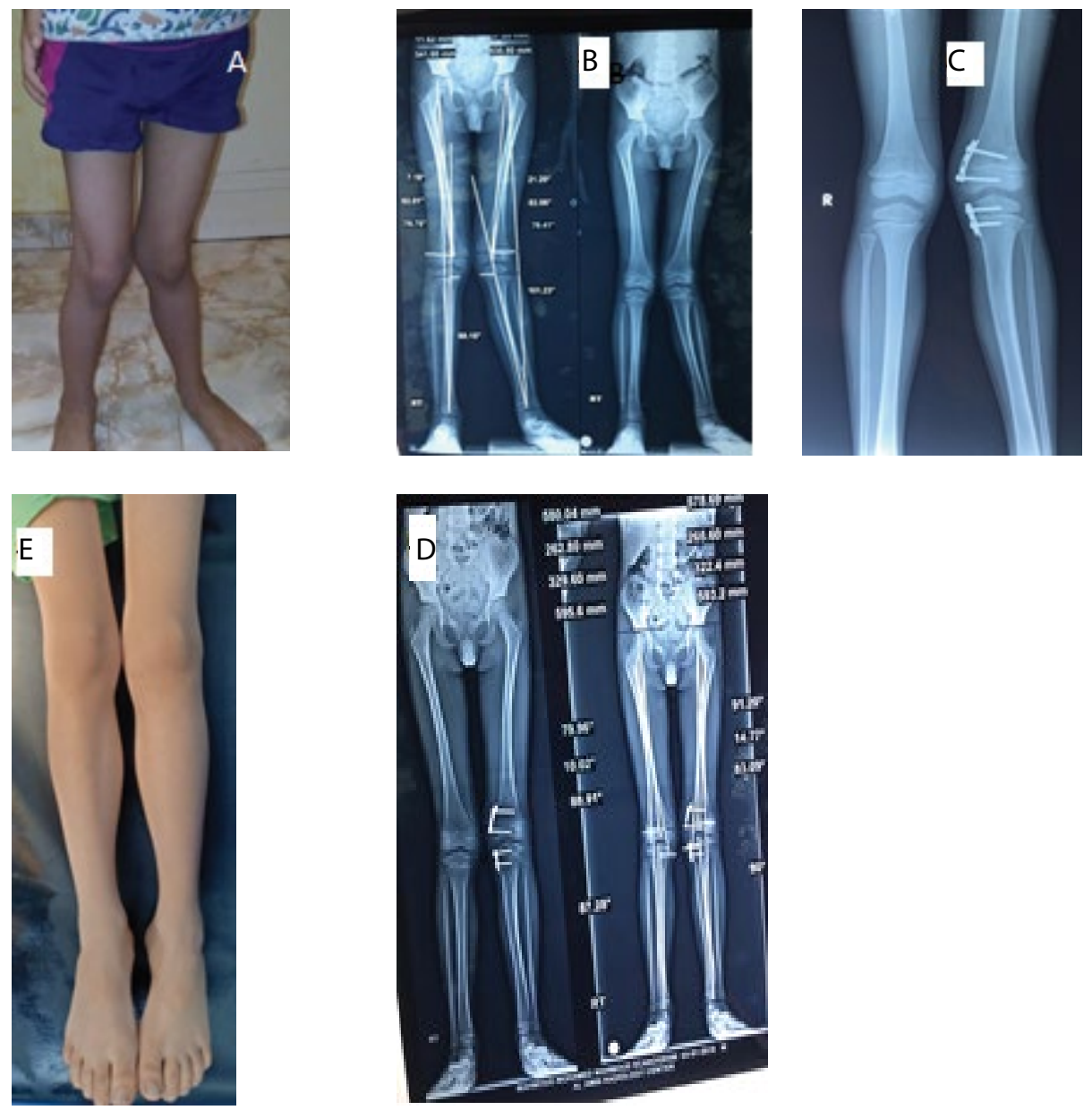

Figure 3: A, preoperative clinical picture of the patient/B ,preoperative scanogram/C, postoperative x-ray / E and D final clinical picture and scanogram for the patient

\section{RESULT}

Between January 2015 and December 2019, twenty eight patients (19 boys 67.8\% and 9 girls $32.2 \%$ ) with 50 valgus knee deformities with twenty two cases with bilateral knee deformity (44 knees $88 \%)$ and six cases with unilateral (6 knees $12 \%$ ) and the mean age of the cases at the time of the operation was 7.8 \pm 2.3 (4-12.5 years).( Tab.1)

Table (1):-Demographic\& clinical characteristic of the studied patients (No.=28).

\begin{tabular}{|c|c|c|c|}
\hline & & Mean \pm SD & Range \\
\hline \multicolumn{2}{|c|}{ Age (years) } & $7.8 \pm 2.3$ & $4-12.5$ \\
\hline \multicolumn{2}{|c|}{ Duration of correction } & $19 \pm 4.6$ & $14-29$ \\
\hline \multirow{2}{*}{\multicolumn{2}{|c|}{ FU (months) }} & $31 \pm 7.8$ & $20-48$ \\
\hline & & No. & $\%$ \\
\hline \multirow[t]{2}{*}{ Sex } & Male & 19 & 67.8 \\
\hline & Female & 9 & 32.2 \\
\hline
\end{tabular}


TREATMENT OF GENU VALGUM IN CHILDREN METHOD OF TEMPORARY HEMIEPIPHYSIODESIS USING EIGHT-PLATES: SHORT-TERM OUTCOME

\begin{tabular}{|c|c|c|c|}
\hline \multirow{2}{*}{ Side } & Bilateral & 22 & 78.6 \\
\cline { 2 - 4 } & Right & 4 & 14.3 \\
\cline { 2 - 4 } & Left & 2 & 7.1 \\
\hline \multirow{3}{*}{ Pathology } & Idiopathic & 11 & 39.3 \\
\cline { 2 - 4 } & Post-rachitic & 12 & 42.9 \\
\cline { 2 - 4 } Site of HEMIEPIPHYSIODESIS & Post traumatic & 5 & 17.9 \\
\cline { 2 - 4 } & MPT+MDF & 6 & 21.4 \\
\hline
\end{tabular}

The mean follow-up was $31 \pm 2.3$ months (range 20-48 months) after plate removal. Two patients reached skeletal maturity at the final follow up. The eight-plates were inserted on the medial distal femur (MDF) in 6 patients (21.4\%) Ten knees (20\%), in proximal tibia (MPT) in $6(21.4 \%)$ nine knees $(18 \%)$ patients and on both femur and tibia $(\mathrm{MPT}+\mathrm{MDF})$ in 16 patients $(57.2 \%)$ Thirty one knees $(62 \%)$.

All patients achieved complete deformity correction after plate removal mean duration 19 \pm 4.6 (14-29 months) and the mean follow up was $31 \pm 7.8$ (20-48 months) and the mean Intermalleolar distances before the procedure was $19.2 \pm 4.3$ changed post to average $1.2 \pm 1$ (Percent of improvement $93.8 \%$ )(significant $p$. value $>0.001$ ) and the mean mLDFA changed from $79.2 \pm 5$ to $87.2 \pm 1.5$ (Percent of improvement 10.1\%) (Significant p. value $>0.001$ ) after the procedure. Neutral mechanical axis with no MAD was the sign of complete correction. Alignment improved in all cases at final follow up and the Radiographic TFA mean changed from $22.3 \pm 4.7$ to $7.8 \pm 4.6$ (Percent of improvement $65.0 \%$ ) (Significant p. value $>0.001$ ) and the Radiographic MPTA changed from $92.6 \pm 3.7$ to $87.8 \pm 0.9$ (Percent of improvement 5.2\%) (Significant p. value > 0.001).(tab. 2)

Table (2): -comparison between pre and post-operative results regarding different parameters of studied patients (No.=28).

\begin{tabular}{|c|c|c|c|c|c|}
\hline & Pre -operative & Post -operative & $\begin{array}{c}\text { Percent of } \\
\text { improvement }\end{array}$ & \multirow{2}{*}{ Paired t test } & \multirow{2}{*}{ p. value } \\
\cline { 2 - 5 } & Mean \pm SD & Mean \pm SD & $\mathbf{( \% )}$ & & $<0.001^{* *}$ \\
\hline Intermalleolar distance & $19.2 \pm 4.3$ & $1.2 \pm 1$ & 93.8 & 29.104 & $<0.001^{* *}$ \\
\hline Radiographic mLDFA & $79.2 \pm 5$ & $87.2 \pm 1.5$ & 10.1 & -9.668 & $<0.001^{* *}$ \\
\hline Radiographic TFA & $22.3 \pm 4.7$ & $7.8 \pm 4.6$ & 65.0 & 26.54 & $<0.001^{* *}$ \\
\hline Radiographic MPTA & $92.6 \pm 3.7$ & $87.8 \pm 0.9$ & 5.2 & 7.737 & \\
\hline
\end{tabular}

In cases when the deformity origin was femoral, (Table: 3 )

Table (3) :-Demographic\& clinical characteristic of patients with femoral deformity (No.=6).

\begin{tabular}{|c|c|c|c|}
\hline & & Mean \pm SD & Range \\
\hline \multicolumn{2}{|c|}{ Age (years) } & $6.7 \pm 2.3$ & $4-10.2$ \\
\hline \multicolumn{2}{|c|}{ Duration of correction } & $17.5 \pm 3.4$ & $14-22$ \\
\hline \multicolumn{2}{|c|}{ FU (months) } & $31.3 \pm 7.1$ & $23-42$ \\
\hline & & No. & $\%$ \\
\hline \multirow[t]{2}{*}{ Sex } & Male & 5 & 83.3 \\
\hline & Female & 1 & 16.7 \\
\hline \multirow{3}{*}{ Side } & Bilateral & 4 & 66.7 \\
\hline & Right & 2 & 33.3 \\
\hline & Left & 0 & 0.0 \\
\hline
\end{tabular}




\begin{tabular}{|c|c|c|c|}
\hline \multirow{3}{*}{ Pathology } & Idiopathic & 0 & 0.0 \\
\cline { 2 - 4 } & Post-rachitic & 4 & 66.7 \\
\cline { 2 - 4 } & Post traumatic & 2 & 33.3 \\
\hline \multirow{3}{*}{ Site of HEMIEPIPHYSIODESIS } & MPT+MDF & 0 & 0.0 \\
\cline { 2 - 4 } & MDF & 6 & 100.0 \\
\cline { 2 - 4 } & MPT & 0 & 0.0 \\
\hline
\end{tabular}

The mean age 6.7 \pm 2.3 (4-10.2) with mean duration of correction 17.5 \pm 3.4 (14-22) and mean follow up duration $31.3 \pm 7.1$ (23-42). Mean mLDFA increased from $73.4 \pm 1.8$ to $86.3 \pm 1.5$ which constitutes an average change 17.6 and when the origin of deformity was tibial, the mean MPTA changed from $87.5 \pm 0.8$ to $87.7 \pm 0.4$. (Fig 5), (Tab. 4).

Table (4): -comparison between pre and post-operative results of patients with femoral deformity (No.=6).

\begin{tabular}{|c|c|c|c|c|c|}
\hline & Pre - operative & Post -operative & Percent of improvement & \multirow{2}{*}{ Paired t test } & \multirow{2}{*}{ p.value } \\
\cline { 2 - 5 } & Mean \pm SD & Mean \pm SD & $(\%)$ & 20.357 & $<0.001^{* *}$ \\
\hline Intermalleolar distance & $15.6 \pm \mathbf{1 . 7}$ & $0.3 \pm \mathbf{0 . 4}$ & 98.1 & -14.946 & $<0.001^{* *}$ \\
\hline Radiographic mLDFA & $73.4 \pm \mathbf{1 . 8}$ & $86.3 \pm \mathbf{1 . 5}$ & 17.6 & 13.373 & $<0.001^{* *}$ \\
\hline Radiographic TFA & $18.3 \pm \mathbf{1 . 2}$ & $6.2 \pm \mathbf{2 . 4}$ & 66.1 & -0.61 & 0.568 \\
\hline Radiographic MPTA & $87.5 \pm \mathbf{0 . 8}$ & $87.7 \pm \mathbf{0 . 4}$ & 0.2 & 0.56 \\
\hline
\end{tabular}

In cases when the deformity origin was Tibial (tab. 5, 6), the mean Intermalleolar distance changed from $15.9 \pm 2.9$ to $0.7 \pm 0.6$ (Percent of improvement 95.6\%) (Significant p. value $>0.001$ ), Radiographic mLDFA changed from $83.6 \pm 6.7$ to $87.6 \pm 0.6$ Radiographic TFA changed from $18.1 \pm 1.8$ to $4.6 \pm 3.2$ (Significant p. value $>0.001$ ) and Radiographic MPTA changed from $94.9 \pm 4.1$ to $88.2 \pm 0.7$ (Significant p. value $>0.001$ ).

Table (5) :-Demographic\& clinical characteristic of patients with tibial deformity (No.=7).

\begin{tabular}{|c|c|c|c|}
\hline & & Mean \pm SD & Range \\
\hline \multicolumn{2}{|l|}{ Age (years) } & $7.9 \pm 2.6$ & $4.2-12.5$ \\
\hline \multicolumn{2}{|c|}{ Duration of correction } & $20.1 \pm \mathbf{3 . 8}$ & $14-25$ \\
\hline \multicolumn{2}{|l|}{ FU (months) } & $31.3 \pm 6.2$ & $26-42$ \\
\hline \multirow{3}{*}{ Sex } & & No. & $\%$ \\
\hline & Male & 3 & 42.9 \\
\hline & Female & 4 & 57.1 \\
\hline \multirow{3}{*}{ Side } & Bilateral & 4 & 57.1 \\
\hline & Right & 1 & 14.3 \\
\hline & Left & 2 & 28.6 \\
\hline \multirow{3}{*}{ Pathology } & Idiopathic & 0 & 0.0 \\
\hline & Post-rachitic & 4 & 57.1 \\
\hline & Post traumatic & 3 & 42.9 \\
\hline \multirow{3}{*}{ Site of HEMIEPIPHYSIODESIS } & MPT+MDF & 0 & 0.0 \\
\hline & MDF & 1 & 14.3 \\
\hline & MPT & 6 & 85.7 \\
\hline
\end{tabular}

Table (6): -comparison between pre and post-operative results of patients with tibial deformity (No. $=7$ ).

\begin{tabular}{|c|c|c|c|c|c|}
\hline & Pre -operative & Post -operative & Percent of improvement & Paired t test & \multirow{2}{*}{ p.value } \\
\cline { 2 - 5 } & Mean \pm SD & Mean \pm SD & $(\%)$ & 16.113 & $<0.001 * *$ \\
\hline Intermalleolar distance & $15.9 \pm \mathbf{2 . 9}$ & $0.7 \pm \mathbf{0 . 6}$ & 95.6 & -1.597 & 0.161 \\
\hline Radiographic mLDFA & $83.6 \pm \mathbf{6 . 7}$ & $87.6 \pm \mathbf{0 . 6}$ & 4.8 & 12.474 & $<0.001 * *$ \\
\hline Radiographic TFA & $18.1 \pm \mathbf{1 . 8}$ & $4.6 \pm \mathbf{3 . 2}$ & 74.6 & 4.755 & $0.003 *$ \\
\hline Radiographic MPTA & $94.9 \pm \mathbf{4 . 1}$ & $88.2 \pm \mathbf{0 . 7}$ & 7.1 & 4.7 \\
\hline
\end{tabular}


No intraoperative complications were recorded. Postoperative superficial infections were reported in one cases (3.57\%) and treated by repeated dressing and IV cephalosporin antibiotics for 10 days and one patient developed limited ROM that improved by extensive physiotherapy, one had lower limb edema postoperative that recover after elevation of the lower limb and application of stockinet. No cases required any osteotomy or repeat the eight-Plate insertion until final follow up. (Table 2)

\section{DISCUSSION}

Genu valgum deformity alter the biomechanics of the knee by causing a distorted stress distribution on the weightbearing surface of the knee joint, and various methods have been proposed to address this problem.

The guided growth methodology by means of transitory hemiepiphysiodesis is a system for amendment of angular deformations of the lower limb in kids (12). The formation of a tie on the convex side of the physis and resulting development from the unarrested side gives correction of the precise distortion. This method is a less invasive surgery accomplished with a low morbidity and can be suspended once the satisfactory alignment is acquired.(13) The idea of the eight-plate lies in the position of a non-rigid extra periosteal plate and two screws, filling in as a central pivot at the edge of the physis.(14)

The purposes of this study were to determine the accuracy of partial hemiepiphysiodesis in correction of genu valgum deformity by evaluating radiological outcome comparing the preoperative and postoperative measurements, the descriptive data on time spent with plate, mean correction time, and determine the safety and reviewing the complications.

In our study we had temporary hemiepiphysiodesis in twenty eight patients (19 boys $67.8 \%$ and 9 girls $32.2 \%$ ) with 50 valgus knee deformities with twenty two cases with bilateral knee deformity ( 44 knees $88 \%$ ) and six cases with unilateral ( 6 knees 12\%) and the mean age at the time of the operation was 7.79 (4-12.5 years). The mean follow-up was 30.75 months (range 20-48 months) after plate removal.

All patients achieved complete deformity correction and the average duration 19 months (14-29 months), the average follow up was 30.75 months (20-48 months), the average Intermalleolar distances before the procedure was $19.2 \mathrm{~cm}$ (12.6-26.5) changed post to average $1.12 \mathrm{~cm}(0-3.6 \mathrm{~cm})$ and the average mLDFA changed from $79.11^{\circ}\left(71.5^{\circ}-88.2^{\circ}\right)$ to $87.26^{\circ}\left(83.6^{\circ}-89^{\circ}\right)$ after the procedure.

The study of Ballal et al. announced 13 patients (28 physes) with genu valgum deformity of mean period of 11.6 years. Patients were treated with the extra-periosteal application of 8 plates. The mean plate time of guided growth was 9.1 months (extend 8-16 months).Femoral genu varum disfigurement (mLDFA) remedied inside $5^{\circ}$ of their ordinary mean qualities, while in tibial genu varum MPTA adjusted inside two degrees of their ordinaryamean qualities, Correction was quicker if the children was under 10 years old. Complication in that study was one patient with plate and screw migration and one patient with deep infection who have surgical debridement but no permanent physeal tethers were encountered after follow up patients between 6 and 32 months after plate removal.(15,16)

The study done by Burghardt et al. checked on guided growth in 10 patients (17 physes) with genu valgum deformity of mean age of 9.7 years utilizing 8 plates. The mean guided growth time was 8.5 months (6-15months). Site of correction were 3 limbs with both distal femur and proximal tibia deformity, 11 limbs with distal femur deformities. Femoral deformity (mLDFA) corrected within three degrees of their ordinary mean values, while MPTA corrected within $2^{\circ}$ of their ordinary mean qualities with failure of correction in two patients. While failure of correction occur in two patients because of no enough growth remaining in the physes.(17)

Casteneda et al. reviewed guided growth in 24 patients ( 52 physes) with genu valgum deformity of mean age of 11.9 years using staples. Site of correction was 18 limbs with both distal femur and proximal tibia deformities, 7 limbs with distal femoral deformities and 9 limbs with proximal tibial deformities. Femoral deformity (mLDFA) corrected

American Research Journal of Orthopedics and Traumatology 
within 60 of their normal mean values, while MPTA corrected within 3o of their normal mean values with exclusion of 5 patients which failure of correction occur. And reviewed failure of correction occur in 5 patients, 5 patients with staple back out and one patient with superficial infection.(18)

Shin et al. reviewed guided growth in 19 (43 physis) patients with genu valgum deformity of mean age of 12.1 years for boys and 10.4 years for girls using staples. Site of correction was distal femur deformities, rebound deformity occur in three distal femoral physis.(19)

El-Batrawy et al in their study in which the use of 43 eight-plates for 22 patients with angular knee joint deformations and recently Zajonz et al in their series that included 198 eight-plates that were embedded close to the knee in 132 children experienced angular knee joint distortions had revealed great outcomes. Both found that guided growth with 8 plate procedure is end up being sheltered and compelling in the treatment of angular knee deformations with advantages over other procedures in children form 3 up to 12 years. $(20,21)$

Niethard applied eight-plates to correct knee joint axial deformities in children with an average age of 9.5 years (range: 2.3-13.7 years). Burghardt studied patients with ages ranging from 4.9 to 13.7 years. No growth plate complications were observed in these studies. $(17,22,23)$

Marangoz archived that there is connection, between the delta joint direction edge and the delta inter-screw angle. This connection isn't identified with the growth pace of the physis. It is an absolutely mathematical correlation, independent of the age and sex of the patient. It very well may be utilized to quantify the anatomical changes of the joint line without the need of full length X-rays. (24)

In this series 3 patients were over the age of 10 years and responded quite well to this technique. All cases in the current series had achieved full correction, but this was the preliminary results in a small sample size.

\section{CONCLUSION}

Temporary hemiepiphysiodesis using eight-Plates is a gentle, simple and effective procedure used to treat genu valgum by modulating growth. Slight overcorrection is desirable due to the rebound phenomenon, especially in young patients with high growth potential and risk groups such as obese children. In adolescents with only low growth potential (older than 14 years), owing to the low correction potential, the indication should be strictly reviewed and the possible failure of therapy should be discussed with the patient family.

\section{REFERENCES}

1. Marc J. Lee MD, Barbara N. Weissman MD, in Imaging of Arthritis and Metabolic Bone Disease, 2009.

2. S.S. Bhise, 2 B. G. Chikhalkar, 3 S. D. Nanandkar, 4G. S. Chavan, 5 Anand P Rayamane Age Determination from of Ossification Center Fusion around Knee Joint in Mumbai Region: A Radiological Study. J Indian Acad Forensic Med. Jan-March 2015, Vol. 37, No. 1.

3. Crenshaw AH J and Perez EA. Diagnosis and management of pediatric genu valgum Campbell's Operative Orthopedics, 11th ed; published by Mosby; 2007; 2985-3070.

4. Kumar A, Gaba S, Sud A, Mandlecha P, Goel L, et al. Comparative study between staples and eight plate in the management of coronal plane deformities of the knee in skeletally immature children. J Child Orthop 2016, 10: 429437.

5. Stevens PM, Klatt JB. Guided growth for pathological physes: radiographic improvement during realignment. J Pediatr Orthop. 2008;28:632-639.

6. Jelinek E, Bittersohl B, Martiny F, et al. The 8-plate versus physeal stapling for temporary hemiepiphyseodesis correcting genu valgum and genu varum: a retrospective analysis of thirty-five patients. International Orthopaedics (SICOT). 2012;36:599-605.doi: 10.1007/s00264-011-1369-5.

American Research Journal of Orthopedics and Traumatology 
7. Stevens,PeterM.M.D.; Maguire,MikeM.D.;Dales,M.D.M.D.;Robins,A.J.M.D.PhysealStapling forIdiopathicGenu Valgum Journal of Pediatric Orthopaedics: September-October 1999 - Volume 19 - Issue 5 - p 645.

8. Arya A, Garg $\mathbf{S}$ and Sinha $\mathbf{J}$ distal femoral varus osteotomy in genu valgum Bone Joint Surg, Vol 91-B, 2009; 206.

9. Watford KE, Jazrawi LM and Eglseder WA Jr. hemiepiphysiodesis of medial femoral in genu valgum. Orthopaedics 2009; 32:1.

10. Burghardt RD, Herzenberg JE. Temporary hemiepiphysiodesis with the eight-plate for angular deformities: mid-term results. J Orthop Sci. 2010;15: 699-704.

11. Burghardt RD, Herzenberg JE, Standard SC, Paley D. Temporary hemiepiphyseal arrest using a screw and plate device to treat knee and ankle deformities in children: a preliminary report. J Child Orthop. 2008; 2:187-97.

12. Zajonz 1. D, Schumann E, Wojan M, Kübler FB, Josten C, Bühligen U, Heyde CE. Treatment of genu valgum in children by means of temporary hemiepiphysiodesis using eight-plates: short-term findings - BMC Musculoskeletal Disorders, 2017,18:456.

13. Stevens PM. Guided growth for angular correction: a preliminary series using a tension band plate 2. - J Pediatr Orthop, 2007, 27:253-259.

14. Ashby E, Eastwood D. Characterization of knee alignment in children with mucopolysaccharidosis 3. types I and II and outcome of treatment with guided growth - J Child Orthop ,2015, 9:227-33.

15. Ballal MS, Bruce CE, Nayagam S. Correcting genu varum and genu valgum in children by guided growth: Temporary hemiepiphysiodesis using tension band plates. J Bone Joint Surg (Br) 2010; 92(2):273- 276.

16. Sprouse C, Tosi L, Stapleton E, Gropman AL, Mitchell FL, Peret R. Musculoskeletal anomalies in a Large cohort of boys with 49, XXXXY - Am J Med Genet C Semin Med Genet, 2013,163C:44-9.

17. Burghardt RD, Specht SC, Herzenberg JE. Mechanical failures of eight-plate guided growth system for temporary hemiepiphysiodesis. J Pediatric Orthop. 2010; 30(6):594-597.

18. Casteneda P, Urquhart B, Sullivan E, Haynes RJ. Hemiepiphysiodesis for the correction of angular deformity about the knee. J Pediatric Orthop.2008; 28:188-191.

19. Shin SJ, cho J, Park MS, et al. Angular deformity correction by asymmetrical physeal suppression in growing children: stapling versus percutaneous transphyseal screw. JPediatric Orthop. 2010; 30 (6):588-593.

20. EIBatrawy Y, Mahran M, EL Gebeily M Temporary Epiphysiodesis In The Correction Of Angular Knee Deformities Using Guided Growth Principle With Eight-Plate. Ain-Shams, University orthopedic journal, $2014 ;(1-7)$.

21. Zajonz 1. D, Schumann E, Wojan M, Kübler FB, Josten C, Bühligen U, Heyde CE. Treatment of genu valgum in children by means of temporary hemiepiphysiodesis using eight-plates: short-term findings-BMC Musculoskeletal Disorders, 2017, 18:456.

22. Bachmann M, Rutz E, Brunner R, Gaston MS, Hirschmann MT, et al. Temporary hemiepiphysiodesis of the distal medial femur: MPFL in danger. Arch Orthop Trauma Surg., 2014 134:1059-1064.

23. Eastwood DM, Sanghrajka AP. Guided growth: recent advances in a deep rooted concept. $J$ Bone Joint Surg.2011;93-B:12-18.doi:10.1302/0301-620X.93B1.25181.

24. Marangoz S, Buyukdogan K, Karahan S.aIs there a correlation between the change in the interscrew angle of the eight-plate and the delta joint orientation angles? Acta Orthop Traumatol Turc , 2017, 51: 39-43.

Citation: Emad Abd-Elhady MD, Mohamed El-Sadek MD, "Treatment of genu valgum in children Method of temporary hemiepiphysiodesis using eight-plates: short-term outcome". American Research Journal of Orthopedics and Traumatology, vol 5, no. 1, 2020, pp. 1-10.

Copyright (c) 2020 Emad Abd-Elhady MD, et al, This is an open access article distributed under the Creative Commons Attribution License, which permits unrestricted use, distribution, and reproduction in any medium, provided the original work is properly cited.

American Research Journal of Orthopedics and Traumatology 